\title{
A search for pre-biotic molecules in hot cores ${ }^{\star}$ (Research Note)
}

\author{
E. S. Wirström ${ }^{1}$, P. Bergman ${ }^{1,2}, \AA$ A. Hjalmarson ${ }^{1}$, and A. Nummelin ${ }^{3}$ \\ 1 Onsala Space Observatory, Chalmers University of Technology, 43992 Onsala, Sweden \\ e-mail: eva@oso.chalmers.se \\ 2 European Southern Observatory, Alonso de Cordova 3107, Vitacura, Casilla 19001 Santiago, Chile \\ 3 Computer science and engineering, Chalmers University of Technology, 41296 Gothenburg, Sweden
}

Received 23 March 2007 / Accepted 11 May 2007

\section{ABSTRACT}

\begin{abstract}
Aims. Our aim is to better understand the complex chemistry of organic molecules in the interstellar medium, leading to the formation of pre-biotic molecules such as amino acids.

Methods. We have performed a search for the pre-biotic molecules amino acetonitrile $\left(\mathrm{H}_{2} \mathrm{NCH}_{2} \mathrm{CN}\right)$ and vinyl acetylene $\left(\mathrm{C}_{2} \mathrm{H}_{3} \mathrm{CCH}\right)$ towards four northern hot core sources using the Onsala $20 \mathrm{~m}$ telescope.

Results. We have determined upper limits to the column density of amino acetonitrile $\left(1-4 \times 10^{13} \mathrm{~cm}^{-2}\right)$ and vinyl acetylene $(2-$ $7 \times 10^{14} \mathrm{~cm}^{-2}$ ) in the observed sources. In addition, from the absence of other lines within the observed frequency band, we have further constrained the column density of oxiranecarbonitrile $\left(c-\mathrm{C}_{3} \mathrm{H}_{3} \mathrm{NO}\right)$ and amino-ethanol $\left(\mathrm{NH}_{2} \mathrm{CH}_{2} \mathrm{CH}_{2} \mathrm{OH}\right)$ in these sources.
\end{abstract}

Key words. ISM: molecules - astrobiology - radio lines: ISM - astrochemistry

\section{Introduction}

One of the theories concerning the origin of life on Earth includes the arrival of important pre-biotic molecules to the surface of the young Earth from space. The mode of delivery is thought to have been a heavy bombardment of comets and meteorites, whose compositions show clear connection to the interstellar medium (ISM) of the pre-solar nebula (Ehrenfreund \& Charnley 2000; Ehrenfreund et al. 2002; Sandford et al. 2006). Interstellar organic molecules are therefore of major interest, both as observational targets (e.g. Hjalmarson et al. 2001) and as constituents of chemical models. For example, since proteins are built from amino acids, the simplest one, glycine $\left(\mathrm{NH}_{2} \mathrm{CH}_{2} \mathrm{COOH}\right)$, has been the object of many observations, laboratory simulations and models of interstellar chemistry.

There has not yet been any unambiguous detection of interstellar glycine, although many searches have been conducted. The tentative detections towards three hot cores reported by Kuan et al. (2003) have been disputed, both by new laboratory measurements and careful analysis by Snyder et al. (2005), as well as by complementary observations towards two of the sources, Sgr B2 and Orion KL (Jones et al. 2007; Cunningham et al. 2007). Even so, the presence of glycine in this type of region is predicted if the amino acid either is formed by reactions in the icy mantles of dust grains, or by gas phase reactions between ice constituents as they evaporate. One of the proposed formation-paths for acids in interstellar ices is the Strecker synthesis, in which water reacts with a nitrile to form the corresponding

* Spectra in FITS format are only available at the CDS via anonymous ftp to cdsarc.u-strasbg.fr (130.79.128.5) or via http://cdsweb.u-strasbg.fr/cgi-bin/qcat?J/A+A/473/177 acid (Peltzer et al. 1984). The nitrile that may form glycine in this way, i.e.

$$
\mathrm{H}_{2} \mathrm{NCH}_{2} \mathrm{CN} \stackrel{2 \mathrm{H}_{2} \mathrm{O}}{\longrightarrow} \mathrm{H}_{2} \mathrm{NCH}_{2} \mathrm{COOH}
$$

is amino acetonitrile $\left(\mathrm{H}_{2} \mathrm{~N}-\mathrm{CH}_{2}-\mathrm{C} \equiv \mathrm{N}\right)$, also known as glycine nitrile. If glycine is formed in interstellar ices in this manner, the amount of amino acetonitrile in gas-phase should be comparable to that of glycine. It even has potential to be more abundant, since Bernstein et al. (2004) have shown that the survival against UV photolysis of amino acetonitrile in interstellar ices is 5 times longer than that of glycine. However, there have been no reported searches for, or detections of, this molecule in the ISM.

The large organic molecule vinyl cyanide $\left(\mathrm{C}_{2} \mathrm{H}_{3} \mathrm{CN}\right)$ has been detected towards several hot cores at abundances up to $7 \times 10^{-9}$ (Ikeda et al. 2001). The increase in abundance with dust temperature suggests that its formation is tightly coupled to the dust, either by formation on the icy grains or by gasphase reactions of evaporated species. Models of the neutral layers in carbon-rich envelopes of evolved stars, with densities and temperatures similar to those of hot cores $\left(n=10^{7} \mathrm{~cm}^{-3}\right.$, $T=300 \mathrm{~K}$ ), have shown that vinyl cyanide does form to some extent by gas-phase reactions (Cernicharo 2004). But according to this model, vinyl acetylene (butenyne), a molecule isoelectronic with vinyl cyanide and containing only carbon and hydrogen $\left(\mathrm{H}_{2} \mathrm{C}=\mathrm{CH}-\mathrm{C} \equiv \mathrm{CH}\right)$, should exist at concentrations two orders of magnitude higher in this kind of environment.

Here we present a dedicated search for amino acetonitrile and vinyl acetylene towards northern hot core sources where the chemically related species have been searched for (glycine) and observed (vinyl cyanide). Frequency coincidences discovered in this single-dish study will need confirmation by interferometric observations of a larger number of lines, before a detection can 
Table 1. Observed source positions.

\begin{tabular}{lccc}
\hline \hline Source & RA $(\mathrm{J} 2000)$ & Dec $(\mathrm{J} 2000)$ & $v_{\text {LSR }}$ \\
\hline Orion KL & $05^{\mathrm{h}} 35^{\mathrm{m}} 14^{\mathrm{s}} .5$ & $-5^{\circ} 22^{\prime} 30^{\prime \prime}$ & +8 \\
W51 e1/e2 & $19^{\mathrm{h}} 23^{\mathrm{m}} 44^{\mathrm{s}} .0$ & $14^{\circ} 30^{\prime} 29^{\prime \prime}$ & +57 \\
S140 & $22^{\mathrm{h}} 19^{\mathrm{m}} 19^{\mathrm{s}} .1$ & $63^{\circ} 18^{\prime} 50^{\prime \prime}$ & -7 \\
W3(OH) & $02^{\mathrm{h}} 27^{\mathrm{m}} 04^{\mathrm{s}} .4$ & $61^{\circ} 52^{\prime} 21^{\prime \prime}$ & -48 \\
\hline
\end{tabular}

be claimed. Detections of amino acetonitrile will enable us to estimate the abundance of its hydrolysis reaction product, glycine. Finding vinyl acetylene will give further clues to the carbon chemistry, possibly leading to formation of amino acids, in hot cores.

\section{Observations}

As a first step towards identifying interstellar amino acetonitrile and vinyl acetylene, we have used the $3 \mathrm{~mm}$ SIS receiver at the Onsala $20 \mathrm{~m}$ telescope to perform a deep search in four sources, namely the Orion KL hot core, the closely spaced molecular cores of W51 e1/e2, and the two circumpolar massive star forming regions $\mathrm{W} 3(\mathrm{OH})$ and $\mathrm{S} 140$ (Table 1). Orion $\mathrm{KL}$ and $\mathrm{W} 51$ e1/e 2 are the two northern sources where the largest column densities of vinyl cyanide were observed by Ikeda et al. (2001), 7 and $5 \times 10^{14} \mathrm{~cm}^{-2}$ respectively. In addition, these are two of the hot cores where Kuan et al. (2003) tentatively identified glycine lines. The third source observed by Kuan et al., Sgr B2, is not observable from the high latitude of Onsala Space Observatory $\left(\sim 57^{\circ}\right)$.

The rotational spectrum of vinyl acetylene from 80 to $165 \mathrm{GHz}$ has been investigated in some detail in the laboratory by Thorwirth \& Lichau (2003), its transitions and molecular parameters are available in the Cologne Database for Molecular Spectroscopy ${ }^{1}$ (CDMS, Müller et al. 2001). The transitions of amino acetonitrile are not, to our knowledge, available in any of the on-line molecular catalogues. However, its rotational spectrum from 9-36 GHz was measured, and its rotational and distortion constants determined, by Brown et al. (1977). From these constants the expected transition frequencies up to $100 \mathrm{GHz}$ were calculated, and the dipole moment of 2.6 D was taken from Pickett (1973).

Observing a $1280 \mathrm{MHz}$ band, $0.8 \mathrm{MHz}$ per channel, centred at about $90.5 \mathrm{GHz}$ during the period November 2003 to April 2004, we managed to cover several interesting transitions from the two targeted molecules. After a small (255 MHz) downward adjustment in centre frequency for the observations run in February-March 2005, another transition was included. In Table 2 we present the observed transitions. In addition, according to recent spectroscopic work by Behnke et al. (2004), at least two transitions of the large cyclic molecule oxiranecarbonitrile $\left(c-\mathrm{C}_{3} \mathrm{H}_{3} \mathrm{NO}\right)$ are covered as well and are included in Table 2. This molecule is a possible precursor of racemic ribose 2,4-diphosphate, an important component of RNA, and can be expected to be present in hot cores (Dickens et al. 1996). To allow us to accurately determine abundance ratios, our study also included observations of a collection of vinyl cyanide transitions around $104 \mathrm{GHz}$, data from the Jet Propulsion Laboratory ${ }^{2}$ catalogue (JPL, Pickett et al. 1998), presented in Table 2.

The observations were made in the beam-switching mode and the system temperature was typically around $400 \mathrm{~K}$. At

\footnotetext{
${ }^{1}$ http://www.ph1.uni-koeln.de/vorhersagen/

${ }^{2}$ http://spec.jpl.nasa.gov/
}

$90 \mathrm{GHz}$ the Onsala $20 \mathrm{~m}$ telescope has a beam $F W H M$ of $\sim 42^{\prime \prime}$ and a main-beam efficiency of $\sim 0.6$.

\section{Results}

\subsection{Vinyl acetylene}

Several transitions of vinyl cyanide were observed towards Orion KL and W51 e1/e2. In the Orion case, the rotation diagram method was used to calculate a rotation temperature and column density, while the lines were too few for this approach in $\mathrm{W} 51 \mathrm{e} 1 / \mathrm{e} 2$. Instead, a rotation temperature of $150 \mathrm{~K}$ was assumed, and the column density was calculated from the highest energy line. These column densities, presented in Table 3, agree well with the ones reported by Ikeda et al. (2001), taking into account the larger beam-size of our observations.

Our observations did not result in detections of vinyl acetylene in any of the observed sources, despite very low noiselevels. Figure 1 presents a part of the observed spectrum towards Orion KL, including the $90.279 \mathrm{GHz}$ vinyl acetylene line, with other line identifications marked. Table 3 presents upper column density limits together with the observed column densities of vinyl cyanide. The upper limits were computed assuming mainbeam peak brightness temperatures corresponding to $3 \sigma$, taken over a $10 \mathrm{~km} \mathrm{~s}^{-1}$ width, corresponding to the measured $F W H M$ of vinyl cyanide in Orion KL.

Comparison between the columns of Table 3 shows that the vinyl acetylene column density at most can be five times larger than that of vinyl cyanide in both Orion KL and W51 e1/e2. In fact, it is not unlikely that the vinyl acetylene column densities are even smaller, resulting from a lower rotation temperature than that assumed above. (E.g. an assumed rotation temperature of $40 \mathrm{~K}$ gives 2.5 times smaller columns.) However, according to the carbon chemistry model developed by Cernicharo (2004) the abundance of vinyl acetylene should be 100 times larger than that of vinyl cyanide. The model failing to reproduce observations by at least a factor of 20 , we conclude that this carbon chemistry model for stellar envelopes does not apply to the typical hot cores where vinyl cyanide has been detected.

\subsection{Amino acetonitrile}

Our observations did not result in a detection of amino acetonitrile. Assuming a rotation temperature of $150 \mathrm{~K}$ and a line-width of $5 \mathrm{~km} \mathrm{~s}^{-1}$, the $3 \sigma$ column density limits of amino acetonitrile were calculated. The results are presented in Table 4.

Recent results of searches for both conformer versions of glycine in Orion KL have resulted in non-detections and $3 \sigma$ upper limits of $3.7 \times 10^{14}$ and $7.7 \times 10^{12} \mathrm{~cm}^{-2}$ for conformer I and II respectively (Cunningham et al. 2007). If the Strecker synthesis in ices is an important formation path for interstellar glycine, the low temperature at which the reactions take place suggests that the main resulting glycine conformer would be the one of lowest energy, conformer I. Furthermore, the amount of glycine in the ISM could not be much larger than that of its dominant precursor, amino acetonitrile. Thus, the upper limit of amino acetonitrile, $1.3 \times 10^{13} \mathrm{~cm}^{-2}$ (Table 4), further constrains the upper limit of glycine conformer I in Orion KL to be a factor of 10 lower than that reached by Cunningham et al. (2007).

In W51 e1/e2, the upper column density limit of amino acetonitrile (Table 4) is found to be considerably lower than the glycine (conformer I) column density calculated from the tentative detection by Kuan et al. (2003), $2.1 \times 10^{14} \mathrm{~cm}^{-2}$, averaged over similar beam size. If a substantial fraction of interstellar 
Table 2. Observed transitions.

\begin{tabular}{lllccc}
\hline \hline \multirow{2}{*}{ Name } & $\begin{array}{c}\text { Molecule } \\
\text { Chemical formula }\end{array}$ & $\begin{array}{l}\text { Frequency } \\
(\mathrm{MHz})\end{array}$ & Transition & $\begin{array}{c}E_{l} \\
(\mathrm{~K})\end{array}$ & $\begin{array}{c}A_{u l} \\
\left(\mathrm{~s}^{-1}\right)\end{array}$ \\
\hline Vinyl acetylene & $\mathrm{C}_{2} \mathrm{H}_{3} \mathrm{CCH}$ & 90279.5 & $10_{0,10} \rightarrow 9_{0,9}$ & 19 & $6.5 \times 10^{-7}$ \\
& & 90675.3 & $10_{2,9} \rightarrow 9_{2,8}$ & 28 & $6.3 \times 10^{-7}$ \\
& & 90802.4 & $10_{4,7 / 6} \rightarrow 9_{4,6 / 5}$ & 55 & $1.1 \times 10^{-6}$ \\
& & 89768.0 & $10_{0,10} \rightarrow 9_{0,9}$ & 20 & $2.7 \times 10^{-5}$ \\
Amino acetonitrile & $\mathrm{H}_{2} \mathrm{NCH}_{2} \mathrm{CN}$ & 90560.3 & $10_{2,9} \rightarrow 9_{2,8}$ & 25 & $2.6 \times 10^{-5}$ \\
& & 89951.5 & $13_{2,11} \rightarrow 12_{2,10}$ & 37 & $3.5 \times 10^{-5}$ \\
Oxiranecarbonitrile & $c-\mathrm{C}_{3} \mathrm{H}_{3} \mathrm{NO}$ & 90493.7 & $13_{1,12} \rightarrow 12_{1,11}$ & 36 & $3.0 \times 10^{-5}$ \\
& & 103575.4 & $11_{0,11} \rightarrow 10_{0,10}$ & 30 & $9.0 \times 10^{-5}$ \\
Vinyl cyanide & $\mathrm{C}_{2} \mathrm{H}_{3} \mathrm{CN}$ & 104212.7 & $11_{2,10} \rightarrow 10_{2,9}$ & 39 & $8.9 \times 10^{-5}$ \\
& & 104419.3 & $11_{6,5 / 6} \rightarrow 10_{6,4 / 5}$ & 108 & $6.5 \times 10^{-5}$ \\
& & 104432.8 & $11_{3,9} \rightarrow 10_{3,8}$ & 50 & $8.5 \times 10^{-5}$ \\
& & 104437.5 & $11_{7,4 / 5} \rightarrow 10_{7,3 / 4}$ & 136 & $5.5 \times 10^{-5}$ \\
& & 104453.9 & $11_{3,8} \rightarrow 10_{3,7}$ & 50 & $8.6 \times 10^{-5}$ \\
& & 104461.5 & $11_{8,3 / 4} \rightarrow 10_{8,2 / 3}$ & 168 & $4.4 \times 10^{-5}$ \\
\hline
\end{tabular}

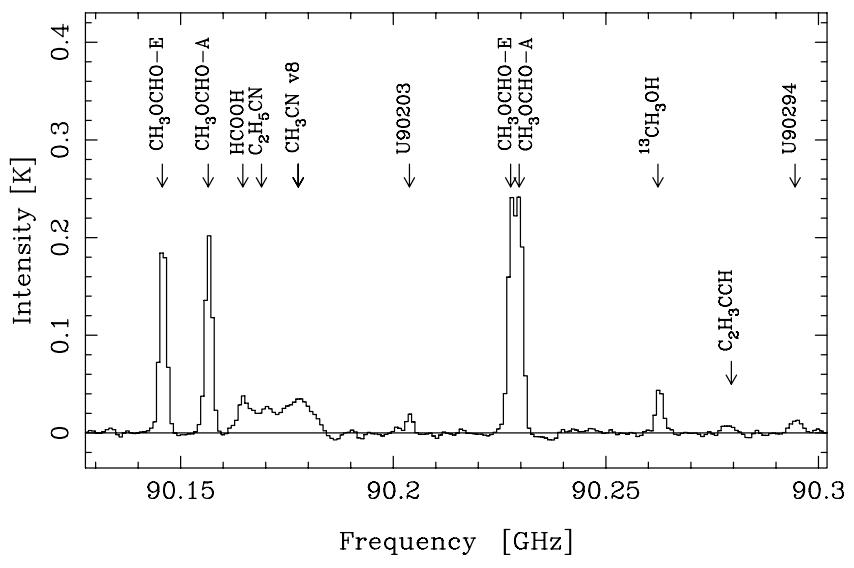

Fig. 1. A part of the observed spectrum towards Orion KL after $31 \mathrm{~h}$ of on-source integration. The noise level is $\sigma=2.7 \mathrm{mK}$. The expected position of one of the vinyl acetylene lines is marked $\left(\mathrm{C}_{2} \mathrm{H}_{3} \mathrm{CCH}\right)$, as well as identifications of other detected lines.

Table 3. Observed, beam averaged column densities of vinyl cyanide $\left(\mathrm{C}_{2} \mathrm{H}_{3} \mathrm{CN}\right)$ and vinyl acetylene $\left(\mathrm{C}_{2} \mathrm{H}_{3} \mathrm{CCH}\right)$.

\begin{tabular}{|c|c|c|c|c|}
\hline \multirow[b]{2}{*}{ Source } & \multicolumn{2}{|c|}{$\mathrm{C}_{2} \mathrm{H}_{3} \mathrm{CN}^{a}$} & \multicolumn{2}{|c|}{${\underline{\mathrm{C}_{2} \mathrm{H}_{3} \mathrm{CCH}^{a}}}^{a}$} \\
\hline & $\begin{array}{l}T_{\mathrm{ex}} \\
(\mathrm{K}) \\
\end{array}$ & $\begin{array}{c}N \\
\left(\mathrm{~cm}^{-2}\right) \\
\end{array}$ & $\begin{array}{l}T_{\mathrm{ex}} \\
(\mathrm{K}) \\
\end{array}$ & $\begin{array}{c}N \\
\left(\mathrm{~cm}^{-2}\right) \\
\end{array}$ \\
\hline Orion KL & 140 & $1.9 \times 10^{14}$ & [150] & $<4.5 \times 10^{14}$ \\
\hline W51 e1/e2 & [150] & $1.6 \times 10^{14}$ & [150] & $<6.8 \times 10^{14}$ \\
\hline S140 & [150] & $<1.0 \times 10^{13}$ & [150] & $<2.4 \times 10^{14}$ \\
\hline W3(OH) & [150] & $<1.3 \times 10^{13}$ & [150] & $<2.1 \times 10^{14}$ \\
\hline
\end{tabular}

${ }^{a}$ Upper limits are taken at $3 \sigma$ noise-level over $10 \mathrm{~km} \mathrm{~s}^{-1}$, assuming the $T_{\text {ex }}$ given in square brackets.

glycine is formed via the Strecker synthesis, as argued above, and there were such large amounts of glycine as reported by Kuan et al. (2003), we would easily have detected amino acetonitrile towards W51 e1/e2.

\subsection{Other limits}

Another suggested formation path for amino acids in interstellar environment is the reaction between protonated amino-alcohols
Table 4. Upper column density limits of amino acetonitrile $\left(\mathrm{H}_{2} \mathrm{~N} \mathrm{CH}_{2} \mathrm{CN}\right)$ and oxiranecarbonitrile $\left(c-\mathrm{C}_{3} \mathrm{H}_{3} \mathrm{NO}\right)$.

\begin{tabular}{lcccc}
\hline \hline \multirow{2}{*}{ Source } & \multicolumn{2}{c}{$\mathrm{H}_{2} \mathrm{NCH}_{2} \mathrm{CN}^{a}$} & & \multicolumn{2}{c}{$\frac{c-\mathrm{C}_{3} \mathrm{H}_{3} \mathrm{NO}^{a}}{T_{\mathrm{ex}}}$} & $\begin{array}{c}N \\
(\mathrm{~K})\end{array}$ & $\left(\mathrm{cm}^{-2}\right)$ & $\begin{array}{c}T_{\mathrm{ex}} \\
(\mathrm{K})\end{array}$ & $\begin{array}{c}N \\
\left(\mathrm{~cm}^{-2}\right)\end{array}$ \\
\hline Orion KL & {$[150]$} & $<1.3 \times 10^{13}$ & {$[40]$} & $<3.4 \times 10^{12}$ \\
W51 e1/e2 & {$[150]$} & $<3.5 \times 10^{13}$ & {$[40]$} & $<9.5 \times 10^{12}$ \\
S140 & {$[150]$} & $<1.1 \times 10^{13}$ & {$[40]$} & $<2.3 \times 10^{12}$ \\
W3(OH) & {$[150]$} & $<1.2 \times 10^{13}$ & {$[40]$} & $<2.3 \times 10^{12}$ \\
\hline
\end{tabular}

${ }^{a}$ Upper limits are taken at $3 \sigma$ noise-level over $5 \mathrm{~km} \mathrm{~s}^{-1}$, assuming the $T_{\text {ex }}$ given in square brackets.

and formic acid $(\mathrm{HCOOH})$, both evaporated from grain surfaces in a hot core environment, forming protonated amino acids (Ehrenfreund \& Charnley 2001). An example is amino-ethanol $\left(\mathrm{NH}_{2} \mathrm{CH}_{2} \mathrm{CH}_{2} \mathrm{OH}\right)$, which would be the precursor of alanine in such a scheme. This molecule has been predicted to be present in detectable amounts in hot cores, but searches towards Orion $\mathrm{KL}$ and W51 e2 have so far not resulted in detections (Widicus et al. 2003).

In our spectrum towards Orion KL, four of the detected emission lines (of which two are U-lines) coincide with aminoethanol $J=9-8$ transitions, all with lower state energies around $340 \mathrm{~K}$ (JPL catalogue). On the other hand, we do not see several lower energy lines with larger A-coefficients, also covered by our spectral range. Of these, the line with highest linestrength (at $89.725 \mathrm{GHz}$ ) gives a $3 \sigma$ upper column density limit of $6.8 \times 10^{13} \mathrm{~cm}^{-2}$, assuming a rotation temperature of $150 \mathrm{~K}$ and a line-width of $5 \mathrm{~km} \mathrm{~s}^{-1}$. Using this column, the inferred intensities of the amino-ethanol transitions coinciding with lines in our spectrum are less than $5 \%$ of the observed ones. Thus, although amino-ethanol does not give rise to any observable lines in our spectrum, the previously reported upper limit of this molecule in Orion KL, $10^{14} \mathrm{~cm}^{-2}$ as reported by Widicus et al. (2003), is somewhat improved. In addition, if amino-ethanol is the main precursor of alanine in hot cores, as outlined by e.g. Ehrenfreund \& Charnley (2001), its abundance limit also sets constraints on the amount of alanine present.

The observations did not result in a detection of oxiranecarbonitrile in any of the observed sources. However, our low $3 \sigma$ upper column density limits, shown in Table 4, further constrain 
its abundance as compared to the previously known limit in Orion KL, derived by Dickens et al. (1996), $6.6 \times 10^{13} \mathrm{~cm}^{-2}$ in a $19^{\prime \prime}$ beam. An upper limit for W51 was also presented there, but the observed core, W51 M, is situated on the $3 \mathrm{~dB}$ contour of our beam which makes a comparison rather pointless.

Acknowledgements. Onsala Space Observatory is the Swedish National Facility for Radio Astronomy, operated by Chalmers University of Technology with financial support from the Swedish Research Council (Vetenskapsrådet). We thank J. H. Black for comments and suggestions.

\section{References}

Behnke, M., Medvedev, I., Winnewisser, M., De Lucia, F. C., \& Herbst, E. 2004, ApJS, 152, 97

Bernstein, M. P., Ashbourn, S. F. M., Sandford, S. A., \& Allamandola, L. J. 2004, ApJ, 601, 365

Brown, R. D., Godfrey, P. D., Ottrey, A. L., \& Storey, J. W. V. 1977, J. Mol. Spectr., 68, 359

Cernicharo, J. 2004, ApJ, 608, L41

Cunningham, M. R., Jones, P. A., Godfrey, P. D., et al. 2007, MNRAS, 376, 1201

Dickens, J. E., Irvine, W. M., Ohishi, M., et al. 1996, Origins of Life and Evolution of the Biosphere, 26, 97
Ehrenfreund, P., \& Charnley, S. B. 2000, ARA\&A, 38, 427

Ehrenfreund, P., \& Charnley, S. B. 2001, in Exo-/Astro-Biology, ed. P. Ehrenfreund, O. Angerer, \& B. Battrick, ESA SP-496, 35

Ehrenfreund, P., Irvine, W., Becker, L., et al. 2002, Rep. Progr. Phys., 65, 1427

Hjalmarson, A., Bergman, P., \& Nummelin, A. 2001, in Exo-/Astro-Biology, ESA SP-496, 263

Ikeda, M., Ohishi, M., Nummelin, A., et al. 2001, ApJ, 560, 792

Jones, P. A., Cunningham, M. R., Godfrey, P. D., \& Cragg, D. M. 2007, MNRAS, 374, 579

Kuan, Y.-J., Charnley, S. B., Huang, H.-C., Tseng, W.-L., \& Kisiel, Z. 2003, ApJ, 593, 848

Müller, H. S. P., Thorwirth, S., Roth, D. A., \& Winnewisser, G. 2001, A\&A, 370, L49

Peltzer, E. T., Bada, J. L., Schlesinger, G., \& Miller, S. L. 1984, Adv. Space Res., 4, 69

Pickett, H. M. 1973, Journal of Molecular Spectroscopy, 46, 335

Pickett, H. M., Poynter, R. L., Cohen, E. A., et al. 1998, J. Quant. Spectr. Radiat. Transf., 60, 883

Sandford, S. A., Aléon, J., Alexander, C. M. O., et al. 2006, Science, 314, 1720

Snyder, L. E., Lovas, F. J., Hollis, J. M., et al. 2005, ApJ, 619, 914

Thorwirth, S., \& Lichau, H. 2003, A\&A, 398, L11

Widicus, S. L., Drouin, B. J., Dyl, K. A., \& Blake, G. A. 2003, in SFChem 2002: Chemistry as a Diagnostic of Star Formation, Proc. a conf. held August 2123, 2002 at University of Waterloo, Waterloo, Ontario, Canada N2L 3G1. ed. C. L. Curry \& M. Fich (Ottawa, Canada: NRC Press), 449 